\title{
SET STABILIZATION OF A CLASS OF POSITIVE SYSTEMS
}

\author{
Lars Imsland* Bjarne A. Foss *,1 \\ * Department of Engineering Cybernetics, \\ Norwegian University of Science and Technology, \\ 7491 Trondheim, Norway. Fax (+47) 73594399 \\ E-mail: \{lars.imsland,bjarne.a.foss\}@itk.ntnu.no
}

\begin{abstract}
A specific class of positive systems is considered, where the system structure allows control of the distribution of "mass" in the system. Some robustness properties of the controller are pointed out, and the applicability of the model class is discussed. An example considering a CSTR modeled by massand energy balances illustrates the presented concepts.
\end{abstract}

Keywords: positive systems, set stabilization, van der Vusse reactor

\section{INTRODUCTION}

When modeling systems for control based on first principles, one often obtains nonlinear ordinary differential equations where the state variables (mass, pressure, level, energy, etc.) are positive. In addition, the control input will also often be positive (valve openings, amount of inflow, heat input, etc.). Hence, the class of positive systems (systems with nonnegative states and inputs) is a natural class of systems to consider in a control setting.

In this paper, we will consider a class of positive systems with strong structural constraints. Some of these constraints are natural from the perspective that the dynamics consist of "mass" flow between the different states, and other constraints are made to ensure controllability under constraints. We will argue that a diverse range of systems can be described by models in this class.

This is further funded by the fact that the intersection between the model class considered herein and the widely studied class of compartmental systems (Jacquez and Simon, 1993) is non-void. The interpretation of states as"masses" of compartments holds for both classes of systems, and similar assumptions (slightly stronger in the case

1 Corresponding author of compartmental systems) concerning the flow of "mass" between the compartments are made. On the other hand, the controllability assumptions made herein, do not have their counterpart in the class of compartmental systems.

However, these controllability assumptions make it possible to specify a controller that controls the distribution of mass for the system class considered herein. The controller is related to the controllers in Bastin and Praly (1999) and De Leenheer and Aeyels (2002), but with distinct differences related to model class and controller specification.

The paper is outlined as follows: In Section 2 the class of systems we look at are specified, while the controller and the convergence result is recapitulated in Section 3. Some robustness properties are pointed out. The applicability of the model class is discussed in Section 4. The example in Section 5 illustrates the use of the controller for a system described by both mass and energy balances.

\section{MODEL CLASS}

We consider positive systems

$$
\dot{x}=f(x, u),
$$


that is, the state is positive $\left(x \in \mathbb{R}_{+}^{n}\right)$, and the input is positive and upper bounded, $u \in U:=$ $\left\{u \in \mathbb{R}_{+}^{m} \mid 0 \leq u_{j} \leq \bar{u}_{j}\right\}$. Each state can be interpreted as the "mass" (amount of material, or some measure of amount) in a "compartment". The controller we will propose exploits system structure, thus we assume the model equations to be on the following form:

$$
f(x, u)=\Phi(x)+\Psi(x)+B(x) u .
$$

Loosely speaking, $\Phi(x)$ represents "interconnection structure" between compartments, $\Psi(x)$ represents uncontrolled external inflows to and outflows from compartments and $B(x) u$ represents controlled external inflows to and outflows from compartments.

Furthermore, we will assume that the state can be divided into $m$ different parts, which will be denoted phases. Phase $j$ will consist of $r_{j}$ states, and have the control $u_{j}$ associated with it, corresponding to either controlled inflow or outflow to compartments of that phase. The states in phase $j$ will be denoted $z^{j}$, such that $x=$ $\left[\left(z^{1}\right)^{\top},\left(z^{2}\right)^{\top}, \ldots,\left(z^{m}\right)^{\top}\right]^{\top}$, and it follows that necessarily, $\sum_{j=1}^{m} r_{j}=n$. Corresponding to this structure, the vector functions $\Phi(x), \Psi(x)$ and the matrix function $B(x)$ are on the form

$$
\begin{aligned}
\Phi(x) & =\left[\phi^{1}(x)^{\top}, \phi^{2}(x)^{\top}, \ldots, \phi^{m}(x)^{\top}\right]^{\top} \\
\Psi(x) & =\left[\psi^{1}(x)^{\top}, \psi^{2}(x)^{\top}, \ldots, \psi^{m}(x)^{\top}\right]^{\top} \\
B(x) & =\operatorname{blockdiag}\left(b^{1}(x), b^{2}(x), \ldots, b^{m}(x)\right) .
\end{aligned}
$$

Note that element $j$ is (in general) a function of $x$, not (only) $z^{j}$. Also note that the partitioning into phases need not be unique.

We will state the assumptions on these functions on the set $D \subseteq \mathbb{R}_{+}^{n}$. In the case of global results, $D=\mathbb{R}_{+}^{n}$.

A1. (Interconnection structure) The function $\Phi: D \rightarrow \mathbb{R}^{n}$ is locally Lipschitz, $\phi_{i}^{j}(x) \geq 0$ for $z_{i}^{j}=0$, and

$$
\sum_{i=1}^{r_{j}} \phi_{i}^{j}(x)=0, j=1, \ldots, m .
$$

A2. (Controlled external flows) The block diagonal matrix function $B(x): D \rightarrow \mathbb{R}^{n \times m}$ is locally Lipschitz and satisfies:

a. Phase $j$ has controlled inflow:

$$
\begin{aligned}
& b_{i}^{j}(x) \geq 0 \text { for all } x \in D \\
& b_{i}^{j}(x)>0 \text { for all } x \in D \text { for at least one } i
\end{aligned}
$$

b. Phase $j$ has controlled outflow:

$$
\begin{aligned}
& b_{i}^{j}(x) \leq 0 \text { for all } x \in D \\
& z_{i}^{j}=0 \Rightarrow b_{i}^{j}(x)=0 \\
& b_{i}^{j}(x)<0 \text { for all } x \in D \text { with } z_{i}^{j} \neq 0
\end{aligned}
$$

The uncontrolled external flows must satisfy some "controllability" assumption in relation to the controlled flows. Before we define this, it is convenient to define the "mass" of each phase, being the sum of the compartment masses of that phase:

$$
M_{j}(x):=\sum_{i=1}^{r_{j}} z_{i}^{j} .
$$

Our control objective will be to control $M_{j}(x)$ to some prespecified desired mass of phase $j$, denoted $M_{j}^{*}$, from initial conditions in $D$. For the control problem to be meaningful, the intersection of the set where $M_{j}(x)=M_{j}^{*}$ and $D$ should be nonempty.

A3. (Uncontrolled external flows) For given $M^{*}=\left[M_{1}^{*}, M_{2}^{*}, \ldots, M_{m}^{*}\right]^{\top}, \Psi(x): D \rightarrow \mathbb{R}^{n}$ is locally Lipschitz and satisfies that $\psi_{i}^{j}(x) \geq$ 0 for $z_{i}^{j}=0$, and in addition, if:

a. Phase $j$ has controlled inflow:

1. For $x \in\left\{x \in D \mid M_{j}(x)>M_{j}^{*}\right\}$, $\sum_{i=1}^{r_{j}} \psi_{i}^{j}(x) \leq 0$ and the set $\{x \in$ $D \mid \sum_{i=1}^{r_{j}} \psi_{i}^{j}(x)=0$ and $\left.M_{j}(x)>M_{j}^{*}\right\}$ does not contain an invariant set.

2. For $x \in\left\{x \in D \mid M_{j}(x)<M_{j}^{*}\right\}$, $-\sum_{i=1}^{r_{j}} \psi_{i}^{j}(x)<\sum_{i=1}^{r_{j}} b_{i}^{j}(x) \bar{u}_{j}$.

b. Phase $j$ has controlled outflow:

1. For $x \in\left\{x \in D \mid M_{j}(x)<M_{j}^{*}\right\}$, $\sum_{i=1}^{r_{j}} \psi_{i}^{j}(x) \geq 0$ and the set $\{x \in$ $D \mid \sum_{i=1}^{r_{j}} \psi_{i}^{j}(x)=0$ and $\left.M_{j}(x)<M_{j}^{*}\right\}$ does not contain an invariant set.

2. For $x \in\left\{x \in D \mid M_{j}(x)>M_{j}^{*}\right\}$, $\sum_{i=1}^{r_{j}} \psi_{i}^{j}(x)<-\sum_{i=1}^{r_{j}} b_{i}^{j}(x) \bar{u}_{j}$.

It is straightforward to confirm that under the above assumptions, $x_{i}=0$ implies $\dot{x}_{i} \geq 0$, that is, the system is positive.

\section{STABILIZING STATE FEEDBACK CONTROLLER}

In this section, the state feedback controller is defined, and a general convergence result is given for a general invariant set $D$ that (is a subset of the set that) A1-A3 hold on. The set $D$ could then be considered a region of attraction.

\subsection{The controller and a convergence result}

As mentioned in the previous section, our control objective is to control the total mass $M_{j}(x)$ of each phase to a prespecified value $M_{j}^{*}$.

To this end, the following constrained, positive state feedback control law is proposed:

$$
u_{j}(x)= \begin{cases}0 & \text { if } \tilde{u}_{j}(x)<0 \\ \tilde{u}_{j}(x) & \text { if } 0 \leq \tilde{u}_{j}(x) \leq \bar{u}_{j} \\ \bar{u}_{j} & \text { if } \tilde{u}_{j}(x)>\bar{u}_{j}\end{cases}
$$

where

$\tilde{u}_{j}(x)=\frac{1}{\sum_{i=1}^{r_{j}} b_{i}^{j}(x)}\left(-\sum_{i=1}^{r_{j}} \psi_{i}^{j}(x)+\lambda_{j}\left(M_{j}^{*}-M_{j}(x)\right)\right)$ 
and $\lambda_{j}$ is a positive constant. Apparently, we can run into situations where the control is not defined if phase $j$ is outflow controlled, since the term $\sum_{i=1}^{r_{j}} b_{i}^{j}(x)$ then might be zero. However, the continuity of the involved functions and the upper bound on the control ensures that the control in these cases unambiguously are defined by $u_{j}(x)=$ $\bar{u}_{j}$.

Define the set

$$
\Omega=\left\{x \in \mathbb{R}_{+}^{n} \mid M_{1}(x)=M_{1}^{*}, \ldots, M_{m}(x)=M_{m}^{*}\right\} .
$$

Assumption 1. There exists a set $D$ that is invariant for the dynamics (1) under the closed loop with control (2), and has a nonempty intersection with $\Omega$.

Assumption 2. For $x \in \Omega \cap D, 0<\tilde{u}_{j}(x)<\bar{u}_{j}$.

Under the given assumptions, the convergence properties of the controller are summarized in the following Theorem, proved in Imsland (2002), see also Imsland and Foss (2002):

Theorem 1. Under the given assumptions, the state of the system (1), controlled with (2) and starting from some initial condition $x(0) \in D$, stays bounded and converges to the set $\Omega \cap D$ which is positively invariant.

To use this theorem, we need to find invariant sets $D$. In some cases, the assumptions hold globally and we can use $D=\mathbb{R}_{+}^{n}$. In other cases, it is possible to choose sets of the shape $D=D_{1}$ or $D=D_{2}$, where

$D_{1}:=\left\{x \in \mathbb{R}_{+}^{n} \mid-\underline{c}_{j} \leq M_{j}(x)-M_{j}^{*} \leq \bar{c}_{j}, j=1, \ldots, m\right\}$ and

$$
\begin{aligned}
& D_{2}:=\left\{x \in \mathbb{R}_{+}^{n} \mid \underline{z}_{j}^{i} \leq z_{j}^{i} \leq \bar{z}_{j}^{i}, i=1, \ldots, r_{j}\right. \text { and } \\
& \left.M_{j}^{*}-\underline{c}_{j} \leq M_{j}(x) \leq M_{j}^{*}+\bar{c}_{j}, j=1, \ldots, m\right\} .
\end{aligned}
$$

For further details and examples, we refer to Imsland (2002).

Note that the convergence result is convergence to the subset $\Omega$, which often (somewhat inaccurate) is referred to as set stability. This does not imply convergence to an equilibrium. However, as pointed out in Imsland (2002) (see also De Leenheer and Aeyels (2002) and Chabour and Kalitine (2002) for similar issues), if the closed loop system has an equilibrium that is asymptotically stable with respect to $\Omega$, this equilibrium will have $D$ as an (estimate of) region of attraction.

\subsection{Discussion of controller}

The controller (2) can be seen as a generalization of the controller in Bastin and Praly (1999). The novelty is threefold:

a) The concept of phases allows to consider systems with multiple inputs. Furthermore, in
Bastin and Praly (1999) the function $\Psi(x)=$ $-A x$ ( $A$ diagonal with nonnegative, at least one positive, diagonal elements) and $B(x)=b$ (a constant nonnegative vector with at least one positive element). Condition A3 (which in this case amounts to A3.a.1) is replaced by the system being zero state detectable through the output $\left[\begin{array}{lll}1 & 1 \ldots 1\end{array}\right] A x$, which has the same effect as A3.a.1. The results of Bastin and Praly (1999) are recently expanded in the direction of single-input compartmental systems in Bastin and Provost (2002).

b) Systems with controlled outflow can be considered.

c) Sufficient conditions are given to allow upper constraints on the input.

\subsection{Robustness}

The proposed feedback scheme is independent of the interconnection structure and hence robust ${ }^{2}$ to model uncertainties in $\Phi(x)$ (as long as Assumption A1 holds). This is the most important robustness property. As mentioned in Bastin and Praly (1999), the interconnection terms are in practical examples often the terms that are hardest to model.

Assume in the rest of this section that the input saturations are not met, that is, $u_{j}(x)=\tilde{u}_{j}(x)$. This will always hold in a neighborhood of $\Omega$. In this case, we can also show some robustness properties with respect to bounded uncertainties in $\Psi(x)$ and $B(x)$.

First note that in the nominal unconstrained case, the feedback (3) linearizes the dynamics of the mass of phase $j$,

$$
\dot{M}_{j}(x)=\lambda_{j}\left(M_{j}^{*}-M_{j}(x)\right) .
$$

We assume further that the modeling errors in $\Psi(x)$ and $B(x)$ are bounded. Mark the "real" values of the terms involved in the controller (3) with a tilde, and assume that there exists normbounded $\Delta_{j}^{\psi}=\Delta_{j}^{\psi}(x, t)$ and $\Delta_{j}^{b}=\Delta_{j}^{b}(x, t)$ (the dependence on $x$ and $t$ is sometimes suppressed for notational simplicity in the following) such that the nominal values (used in the controller) are related to the real values as

$$
\begin{aligned}
\sum_{i=1}^{r_{j}} \tilde{\psi}_{j}^{i}(x) & =\sum_{i=1}^{r_{j}} \psi_{j}^{i}(x)+\Delta_{j}^{\psi}(x, t), \\
\sum_{i=1}^{r_{j}} \tilde{b}_{j}^{i}(x) & =\left(1+\Delta_{j}^{b}(x, t)\right) \sum_{i=1}^{r_{j}} b_{j}^{i}(x) .
\end{aligned}
$$

The real dynamics of phase $j$ can then be written

2 Robust in the sense that convergence to $\Omega$ still holds. Note that changes in $\Phi(x)$ will typically move the equilibria on $\Omega$. 


$$
\begin{aligned}
\dot{M}_{j}(x)= & \sum_{i=1}^{r_{j}} \tilde{\psi}_{j}^{i}(x)+\sum_{i=1}^{r_{j}} \tilde{b}_{j}^{i}(x) u_{j}(x) \\
= & \lambda_{j}\left(M_{j}^{*}-M_{j}(x)\right)+\Delta_{j}^{\psi} \\
& +\Delta_{j}^{b}\left(-\sum_{i=1}^{r_{j}} \psi_{i}^{j}(x)+\lambda_{j}\left(M_{j}^{*}-M_{j}(x)\right)\right)
\end{aligned}
$$

The last part is in general not bounded in terms of $x$. However, we assume that we can define

$$
\begin{array}{r}
\delta_{j}(t)=\Delta_{j}^{\psi}(x(t), t)+\Delta_{j}^{b}(x(t), t)\left(-\sum_{i=1}^{r_{j}} \psi_{i}^{j}(x(t))\right. \\
\left.+\lambda_{j}\left(M_{j}^{*}-M_{j}(x(t))\right)\right)
\end{array}
$$

such that $\delta_{j}(t)$ is norm-bounded, $\delta_{j}(t) \leq \bar{\delta}_{j}$. This requires either that $\Delta_{j}^{b}(x(t), t) \equiv 0$, or that we know that $x(t)$ is bounded (which is guaranteed by initial conditions in a bounded, invariant set).

The mass dynamics can under the above assumptions be written

$$
\dot{M}_{j}(x(t))=-\lambda_{j}\left(M_{j}(x(t))-M_{j}^{*}\right)+\delta_{j}(t) .
$$

Since this is linear, it is easy to solve this to find

$$
\begin{array}{r}
M_{j}(x(t))=M_{j}^{*}+e^{-\lambda_{j}\left(t-t_{0}\right)}\left(M_{j}\left(x\left(t_{0}\right)\right)-M_{j}^{*}\right) \\
+\int_{t_{0}}^{t} e^{-\lambda_{j}(t-\tau)} \delta_{j}(\tau) d \tau
\end{array}
$$

where the last element is bounded,

$$
\left|\int_{t_{0}}^{t} e^{-\lambda_{j}(t-\tau)} \delta_{j}(\tau) d \tau\right| \leq \frac{1-e^{-\lambda_{j}\left(t-t_{0}\right)}}{\lambda_{j}} \bar{\delta}_{j} \leq \frac{\bar{\delta}_{j}}{\lambda_{j}} .
$$

We see that $M_{j}(x(t))$ converges to the set $\left\{M_{j}|| M_{j}-\right.$ $\left.M_{j}^{*} \mid \leq \frac{\bar{\delta}_{j}}{\lambda_{j}}\right\}$ which can be made arbitrarily close to $M_{j}^{*}$ by choosing $\lambda_{j}$ large. Of course, in choosing $\lambda_{j}$ large, the system might become more vulnerable to the influence of measurement noise and unmodeled dynamics.

The above analysis is only valid as long as the input is not saturated. What happens when the input is saturated can be (conservatively) analyzed by examining if the "Lyapunov function" of Theorem 1 is still decreasing under the allowed perturbations. This can be done by checking if assumptions similar to Assumption A3 hold for the perturbed flows.

\section{APPLICABILITY OF THE MODEL CLASS}

The system class (1) and accompanying control design method has wide applicability. Referring to Imsland (2002) the class has been applied to a number of different examples. We will briefly summarize these results in the following.

- A system comprised of three tanks in series (three states) was investigated using either the inflow to the first tank, the outflow from the third tank, or both as control input(s). In the one control input cases the total mass in the three tanks was controlled. In the two control input case the masses in tank one, and tank two and three; or the masses in tank one and two, and tank three were controlled. Convergence from non-local regions (in one case globally) to a stable equilibrium was shown in all cases.

A compartmental description of the three tanks would typically consist of three compartments, each linked to one tank. The difference to the phase notion is apparent. In the one control input cases, the total mass is controlled meaning that the single phase consists of the masses of the three tanks. In the two control input case the masses in one tank and the two other tanks, respectively, define the phases.

The "interconnection structure" $\Phi(x)$ includes the internal flows between the tanks in the one control input case while it includes the internal flow between the two tanks within one phase in the two control input case. The uncontrolled external flows $\Psi(x)$ include the flow between the two phases, i.e. between the two first tanks or the two last tanks, in the two control input case. In the one control input case $\Psi(x)$ consists of the inflow to the first tank when the control input is defined by the outflow from the third tank, or vice versa. The robustness with respect to modeling errors in $\Phi(x)$ and $\Psi(x)$ is obviously important since these terms will contain errors.

- An 2-dimensional food-chain (prey-predator) system (Ortega et al., 1999) with one control input corresponding to the creation of prey has been examined applying the controller (2). The (one) phase was defined by the total mass, of prey and predator, in the system. Global convergence to an asymptotically stable equilibrium was shown. The system can be generalized to an $n$-dimensional food-chain (prey-predators) system again using the total mass, of prey and predators, in the system as the controlled variable. The controller guarantees convergence to $\Omega$, and simulations show convergence to the single desired equilibrium on $\Omega$, but Lyapunovbased analysis of the dynamics of $\Omega$ did, however, not succeed in this case.

- Gas-lifted wells are important as a means to produce oil and gas from hydrocarbon reservoirs with low reservoir pressure (Golan and Whitson, 1991). The well system consist of two volumes: volume 1 holding gas, and volume 2 holding oil and gas. The system is divided into two phases, the mass of gas in the two volumes and the mass of oil in volume 2. The two control inputs are the gas inflow to volume 1 , and the gas and oil outflow from volume 2. Analysis on a 3-dimensional model showed local convergence to an asymptotically stable equilibrium on $\Omega$. Further, simulations using the controller on an industry-standard simulator (Bendiksen et al., 1991), gave nice results.

- It should be noted that convergence to $\Omega$ does not necessarily imply convergence to an equilibrium. This was shown on a synthetic 3dimensional system where the analysis indicated 
and simulations showed convergence to a periodic orbit in $\Omega$.

The theory has also been applied to a standard test case in process control, the Van der Vusse reactor. Details on this are given in the next section.

\section{EXAMPLE: VAN DER VUSSE REACTOR}

We consider the van der Vusse reaction kinetic scheme

$$
\begin{gathered}
A \rightarrow B \rightarrow C \\
2 A \rightarrow D
\end{gathered}
$$

taking place in a CSTR. Application of the controller on this reactor based on a mass balance model was demonstrated in Imsland (2002). Here, we will use a model consisting of both mass and energy balance (the two phases) taken from Chen et al. (1995), and control heat removal, and inflow rate of substance $A$.

The first phase consist of a mass balance of substance $A$ and $B$, on a concentration $\left(c_{A}\right.$ and $\left.c_{B}\right)$ basis. The second phase consists of an energy balance that describes the cooling that is caused by the cooling jacket. The states are the temperatures in the reactor, $T$ and in the cooling jacket, $T_{K}$. Energy is removed from the cooling jacket by means of a heat exchanger. The rate of energy removal is the second input to the system. The mass and energy balance constitutes the two phases according to the setup in Section 2, the first phase being inflow controlled, the second outflow controlled. The model taken from Chen et al. (1995) is

$$
\begin{aligned}
\dot{c}_{A} & =-k_{1}(T) c_{A}-k_{3}(T) c_{A}^{2}+u_{1}\left(c_{A f}-c_{A}\right) \\
\dot{c}_{B} & =k_{1}(T) c_{A}-k_{2}(T) c_{B}-u_{1} c_{B} \\
\dot{T} & =u_{1}\left(T_{0}-T\right)-\frac{\Delta E_{R}(x)}{\rho C_{p}}+\frac{k_{w} A_{R}}{\rho C_{p} V_{R}}\left(T_{K}-T\right) \\
\dot{T}_{K} & =\frac{1}{m_{K} C_{P K}}\left(-u_{2}+k_{w} A_{R}\left(T-T_{K}\right)\right)
\end{aligned}
$$

where

$$
\begin{aligned}
& \Delta E_{R}(x)=k_{1}(T) c_{A} \Delta H_{R_{A B}} \\
& \quad+k_{2}(T) c_{B} \Delta H_{R_{B C}}+k_{3}(T) c_{A}^{2} \Delta H_{R_{A D}}
\end{aligned}
$$

and the reaction kinetics are given from the Arrhenius law

$$
k_{i}(T)=k_{i 0} e^{E_{i} / T}, i=1,2,3 .
$$

Nominal values of the physical and chemical parameters in the model (6) can be found in Chen et al. (1995).

Since the reactor and the cooling jacket have different heat capacities, the transfer of energy between them leads to asymmetric temperature changes. This means that the energy transfer does not fulfill the interconnection assumption A1. This is remedied by taking the energies $\rho C_{p} V_{R} T$ and $m_{K} C_{P K} T_{K}$ as states, in stead of the temperatures.
The control problem (from Chen et al. (1995)) is to stabilize the system at the working point $c_{A}=2.14 \frac{\mathrm{mol}}{\mathrm{l}}, c_{B}=1.09 \frac{\mathrm{mol}}{\mathrm{l}}, T=387.2 \mathrm{~K}$ and $T_{K}=386.1 K$.

The input is then defined in terms of (2) and

$$
\begin{aligned}
& \tilde{u}_{1}=\frac{1}{c_{A f}-c_{A}-c_{B}}\left(k_{3}(T) c_{A}^{2}\right. \\
& \left.\quad+k_{2}(T) c_{B}+\lambda_{1}\left(M_{1}^{*}-M_{1}(x)\right)\right) \\
& \tilde{u}_{2}=u_{1}\left(T_{0}-T\right) \rho C_{p} V_{R} \\
& \left.\quad-V_{R} \Delta E_{R}(x)-\lambda_{2}\left(M_{2}^{*}-M_{2}(x)\right)\right),
\end{aligned}
$$

with saturations at $\bar{u}_{1}=35 \frac{1}{h}$ and $\bar{u}_{2}=9000 \frac{\mathrm{kJ}}{\mathrm{h}}$. The phase masses are $M_{1}(x)=c_{A}+c_{B}$ and $M_{2}(x)=\rho C_{p} V_{R} T+m_{K} C_{P K} T_{K}$.

The "controllability Assumption" A3 for the first phase holds (at least) for $0 \leq c_{A}+c_{B} \leq 7$, for a reasonable temperature range. For the second phase, $\mathrm{A} 3^{3}$ holds only for a rather small operating range around the desired equilibrium. The reason for this is related to the exothermic nature of the reaction - for some initial conditions close to the desired equilibrium, the energy produced by the reaction is larger than the cooling jacket capacity, such that the total energy is increasing. A remedy for getting a larger guaranteed region of attraction could be to choose another equilibrium, with lower temperatures. This could also be seen as choosing an operating point with better controllability. However, simulations indicate that the controller still works well even outside the region where the controllability assumptions for the second phase holds, since the system dynamics take the states into a region where the assumption holds. This illustrates the sufficient nature of A3.
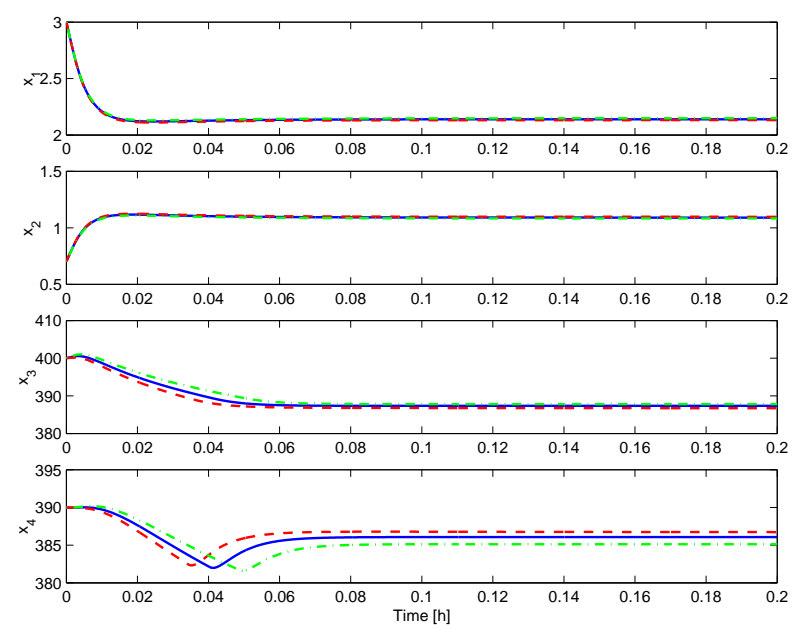

Fig. 1. Simulation of Van der Vusse reactor showing the states, from initial condition $c_{A}=3.0$, $c_{B}=.70, T=400$ and $T_{K}=390$. Nominal parameter set is shown with whole lines, set 1 is dashed, set 2 is dash-dotted.

The simulations in Figures 1-3 show that the controller is robust to the two "extreme" cases of parameter uncertainty taken from Chen et al.

\footnotetext{
3 Note that the input $u_{1}$ in (6c) is taken as a function of state while checking A3 for phase 2.
} 

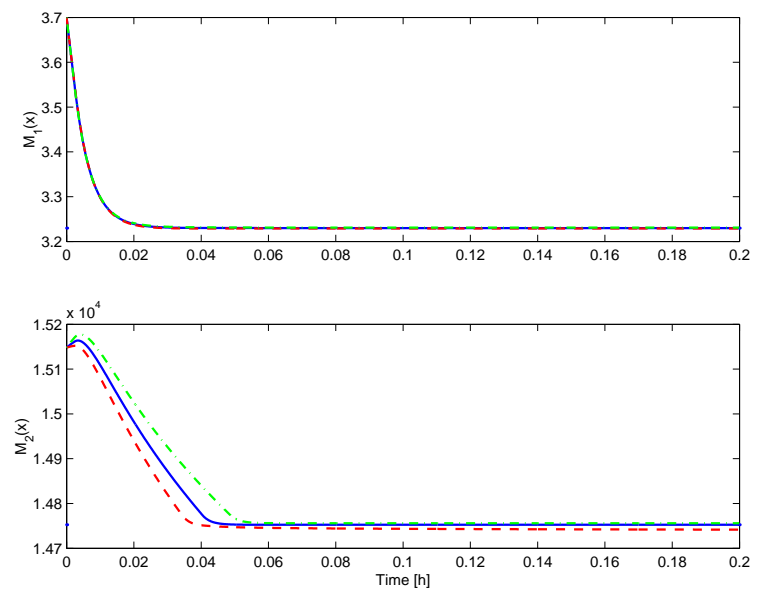

Fig. 2. Simulation of Van der Vusse reactor showing the masses of the phases. Nominal parameter set is shown with whole lines, set 1 is dashed, set 2 is dash-dotted.
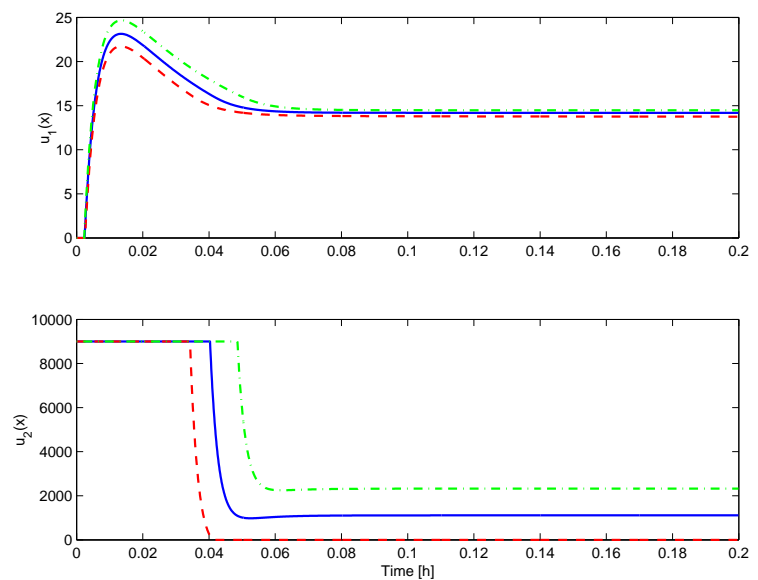

Fig. 3. Simulation of Van der Vusse reactor showing the inputs. Nominal parameter set is shown with whole lines, set 1 is dashed, set 2 is dash-dotted.

(1995) in the sense that stability and convergence to close to $\Omega$ is preserved. However the desired equilibrium is only approximately preserved. Note that for parameter set 2 , the second input reaches its upper saturation at convergence, such that the theory does not really cover this case. Physically, the saturation says that heat removal is not necessary at this working point, for these parameters. Also the controller in Chen et al. (1995) saturates at the equilibrium for this parameter set.

Since the "mass" of phase 2 is increasing initially (Figure 3), the controllability assumptions A3 are not fulfilled for these initial conditions. The controller still works well, as discussed above.

\section{DISCUSSION AND CONCLUDING REMARKS}

The system class is potentially advantageous to systems with positive state variables. Positive state variables are common in dynamic model based on first principles. The advantage is pronounced for systems with an internal structure that is susceptible to the presented system class and that are hard to model accurately. We have presented several quite different examples of such systems.

An obvious limitation of the this paper is the requirement of state feedback control. The natural approach to the output feedback problem is in this case to use observers to estimate the state. Design of observers that can exploit positivity and system structure in a similar manner as the feedback design, is an interesting area for further research.

To conclude we have presented a system class for positive systems, an accompanying state feedback controller with robust stability guarantees, and argued that the theory has potentially wide applicability.

\section{REFERENCES}

Bastin, Georges and Agnès Provost (2002). Feedback stabilisation with positive control of dissipative compartmental systems. In: Proceedings of MTNS.

Bastin, Georges and Laurent Praly (1999). Feedback stabilisation with positive control of a class of dissipative mass-balance systems. In: Proceedings of the 14th IFAC World Congress, Beijing, P. R. China.

Bendiksen, Kjell H., Dag Malnes, Randi Moe and Sven Nuland (1991). The dynamic two-fluid model OLGA: Theory and application. SPE Production Engineering pp. $171-180$.

Chabour, Rachid and Boris Kalitine (2002). Semi-definite Lyapunov functions - stability and stabilizability. IEEE Trans. Aut. Control. To appear.

Chen, Hong, Andreas Kremling and Frank Allgöwer (1995). Nonlinear predictive control of a benchmark CSTR. In: Proc. 3rd European Control Conference ECC'95. pp. 3247-52.

De Leenheer, Patrick and Dirk Aeyels (2002). Stabilization of positive systems with first integrals. Automatica 38(9), 1583-1589.

Golan, Michael and Curtis H. Whitson (1991). Well Performance. 2nd ed.. Prentice-Hall.

Imsland, Lars and Bjarne A. Foss (2002). A state feedback controller for a class of positive systems: Application to gas lift stabilization. Submitted to European Control Conference, 2003, Cambridge, England.

Imsland, Lars Struen (2002). Topics in Nonlinear Control - Output Feedback Stabilization and Control of Positive Systems. PhD thesis. Norwegian University of Science and Technology, Department of Engineering Cybernetics.

Jacquez, John A. and Carl P. Simon (1993). Qualitative theory of compartmental systems. SIAM Rev. 35(1), 43-79.

Ortega, Romeo, Alessandro Astolfi, Georges Bastin and Hugo Rodriguez-Cortes (1999). Output feedback control of food-chain systems. In: New Directions in Nonlinear Observer Design (H. Nijmeier and T.I Fossen, Eds.). pp. 291 - 310. Lecture Notes in Control and Information Sciences. Springer Verlag. 